\title{
Auditory Processing Assessment in Children with Attention Deficit Hyperactivity Disorder: An Open Study Examining Methylphenidate Effects
}

\author{
Bianca Pinheiro Lanzetta-Valdo ${ }^{1}$ Giselle Alves de Oliveira ${ }^{2}$ Jane Tagarro Correa Ferreira ${ }^{2}$ \\ Ester Miyuki Nakamura Palacios ${ }^{2}$
}

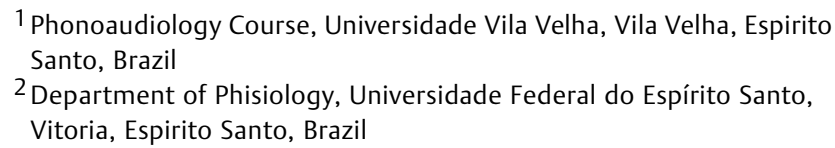

${ }^{2}$ Department of Phisiology, Universidade Federal do Espírito Santo, Vitoria, Espirito Santo, Brazil

Address for correspondence Bianca Pinheiro Lanzetta-Valdo, MSc, Phonoaudiology Course, Vila Velha University, Rua Luís José, 21 Vila Velha, Espirito Santo 29102-920, Brazil (e-mail: bianca.lanzetta.valdo@gmail.com).

Int Arch Otorhinolaryngol 2017;21:72-78.

\begin{abstract}
Keywords

- auditory processing disorder

- child

- methylphenidate

- auditory perceptual disorders

Introduction Children with Attention Deficit Hyperactivity Disorder can present Auditory Processing (AP) Disorder.

Objective The study examined the AP in ADHD children compared with non-ADHD children, and before and after 3 and 6 months of methylphenidate (MPH) treatment in ADHD children.

Methods Drug-naive children diagnosed with ADHD combined subtype aging between 7 and 11 years, coming from public and private outpatient service or public and private school, and age-gender-matched non-ADHD children, participated in an open, non-randomized study from February 2013 to December 2013. They were submitted to a behavioral battery of AP tests comprising Speech with white Noise, Dichotic Digits (DD), and Pitch Pattern Sequence (PPS) and were compared with non-ADHD children. They were followed for 3 and 6 months of MPH treatment $(0.5 \mathrm{mg} / \mathrm{kg} /$ day $)$.

Results ADHD children presented larger number of errors in DD $(p<0.01)$, and less correct responses in the PPS $(p<0.0001)$ and in the SN $(p<0.05)$ tests when compared with non-ADHD children. The treatment with $\mathrm{MPH}$, especially along 6 months, significantly decreased the mean errors in the DD $(p<0.01)$ and increased the correct response in the PPS $(p<0.001)$ and SN $(p<0.01)$ tests when compared with the performance before MPH treatment.

Conclusions ADHD children show inefficient AP in selected behavioral auditory battery suggesting impaired in auditory closure, binaural integration, and temporal ordering. Treatment with MPH gradually improved these deficiencies and completely reversed them by reaching a performance similar to non-ADHD children at 6 months of treatment.
\end{abstract}

\section{Introduction}

Attention Deficit Hyperactivity Disorder (ADHD) is a neurobiological condition characterized by symptoms of inattention, hyperactive, and impulsive behaviors. ${ }^{1}$ It is the most common psychiatry disorder with 5.3\% prevalence in school children population ${ }^{2}$ and high association with comorbidities, such as Oppositional Defiant Disorder (ODD). ${ }^{3}$

Auditory Processing (AP) refers to the perceptual processing of auditory information in the Central Nervous System (CNS), received

October 12, 2015

accepted

November 6, 2015

published online

March 31, 2016
DOI http://dx.doi.org/

10.1055/s-0036-1572526. ISSN $1809-9777$.
Copyright $(2017$ by Thieme-Revinter

Publicações Ltda, Rio de Janeiro, Brazil
License terms

(c) $(1) \$$ 
including sound localization, auditory discrimination, auditory pattern recognition, temporal aspects of hearing during exposure to competitive, and degraded acoustic signs. ${ }^{4}$

Auditory Processing Disorder (APD) refers to auditory perceptual difficulties that are not related to peripheral hearing deficits or language and cognitive dysfunctions. APD children may have difficulties hearing in noisy environments, understanding instructions, ${ }^{4}$ reading, and spelling, as well as poor concentration and impaired memory. ${ }^{5}$ Disorders such as ADHD, autism, and learning disabilities may present similar symptoms. ${ }^{6}$

Recently, studies have suggested that ADHD children show sensory processing deficits, in which the underlying pathophysiology is poorly understood. ${ }^{7}$ Although APD and ADHD have overlapping clinical characteristics, they are distinct entities, requiring accurate diagnoses and appropriate interventions. ${ }^{8}$ Stimulant drugs, such as methylphenidate (MPH), are the standard pharmacological treatment for ADHD. ${ }^{9}$ According to Ozdag et $\mathrm{al}^{10}{ }^{10} \mathrm{MPH}$ may improve the deficits of auditory information processing in ADHD children with no influence over information inputs. ${ }^{8}$ However, MPH effect data on the auditory processing in ADHD children are scarce. To better understand this issue, we proposed analyzing the auditory processing, through a behavioral auditory battery, in ADHD children before and after MPH treatment. Our hypothesis was that ADHD children would present deficits in the discrimination of noise environmental, of temporal processing, and of auditory attention, when compared with non-ADHD children, and that these deficits would improve along 6 months of MPH treatment.

The study examined the AP in ADHD children compared with non-ADHD children, and before and after 3 or 6 months of methylphenidate (MPH) treatment in ADHD children.

\section{Methods}

\section{Subjects}

Children from both genders, aged from 7 to 11 years, which met criteria for ADHD combined subtype diagnosis with no previous pharmacological treatment, were included in this open, nonrandomized study. From February 2013 to December 2013, patients were sequentially included as they were referred to a specialized private outpatient service or public outpatient service in the Medical School Hospital. The control group was comprised of non-ADHD children with similar age, gender, and scholar degree enrolled in public and private schools within the same city from October 2013 to December 2013.

Ethical approval was provided by the Brazilian Institutional Review Board at the Federal University, Brazil, under registration number 194.208, which was conducted in strict adherence to the Declaration of Helsinki and in accordance with ethical standards of the Committee on Human Experimentation.

\section{Inclusion Criteria}

To qualify for inclusion, subjects of control and ADHD groups had to meet the following criteria ${ }^{1}$ : age from 7 to 11, right handed, able to read and write in Portuguese,
Raven's Progressive Matrices scores at or above 5th percentile, without history of clinical, pshyatric ou neurological disorders except Oppoitional Defiant Disorder (ODD), no treatment with psychotropic drugs in the last six months and normal audiometric thresholds.

\section{Exclusion Criteria}

The exclusion criteria were otological disease, learning disabilities, communication difficulties, neurological and psychiatric commodities, Raven's Standard Progressive Matrices scores below 5 th percentil and music abilities.

\section{Experimental Design}

The general experimental protocol is schematized in - Fig. 1 . Children were first assessed in specialized outpatient services for screening of inclusion and exclusion criteria. If they were eligible, they received an invitation to participate in the study.

After written informed consent has been signed, children, parents (or caregivers), and teachers underwent a diagnostic assessment for ADHD and subtypes, using Portuguese versions of DSM-IV-TR criteria. ${ }^{1}$ A structured clinical interview was conducted with parents and school teachers through SNAP IV ${ }^{11}$ and Conners Rating Scales Revised. ${ }^{12}$ Subsequently, children were evaluated by physical and neurological examinations and referred to visual, auditory (audiometry, tympanometry and Acoustic Reflex Test), and biochemical exams. Finally, they were forwarded to the Hearing Health Program, where they underwent a behavioral battery of AP tests before and after 3 and 6 months of MPH treatment $(0.5 \mathrm{mg} / \mathrm{kg} /$ day, twice day).

\section{Auditory Processing Assessment}

Before the battery tests, the same examiner systematically conducted a brief training according to the description below.

\section{Speech with White Noise Test (SN)}

This test evaluates the ability to identify monosyllabic words presented at $40 \mathrm{~dB}$ HL, with $5 \mathrm{~dB}$ above white noise intensity to each ear. Children were instructed to repeat the words which they understood regardless of background noise. The SN test assays the selective auditory attention and auditory closure (ability to understand a message whether incomplete) and is measured by number of correct responses. ${ }^{13}$

\section{Dichotic Digits (DD)}

The test consists of 20 sequences of 4 digits, ranging from one to nine (except numbers two, three and six) which represent dissyllable of English Language (Brazilian Portuguese version). Two digits (50 dB HL) are presented simultaneously to each ear in a binaural integration task. The child was instructed to respond the four numbers presented regardless of order. ${ }^{13}$ This test is very sensitive to auditory processing problems and measured by number of errors. ${ }^{4}$

\section{Pitch Pattern Sequence (PPS)}

The PPS test, ${ }^{14}$ children version, requires discriminations of frequency pattern and temporal ordering of nonverbal 
- 7-11 years old

- Male or Female

- Strict criteria for inclusion and exclusion

\section{Clinical screening}

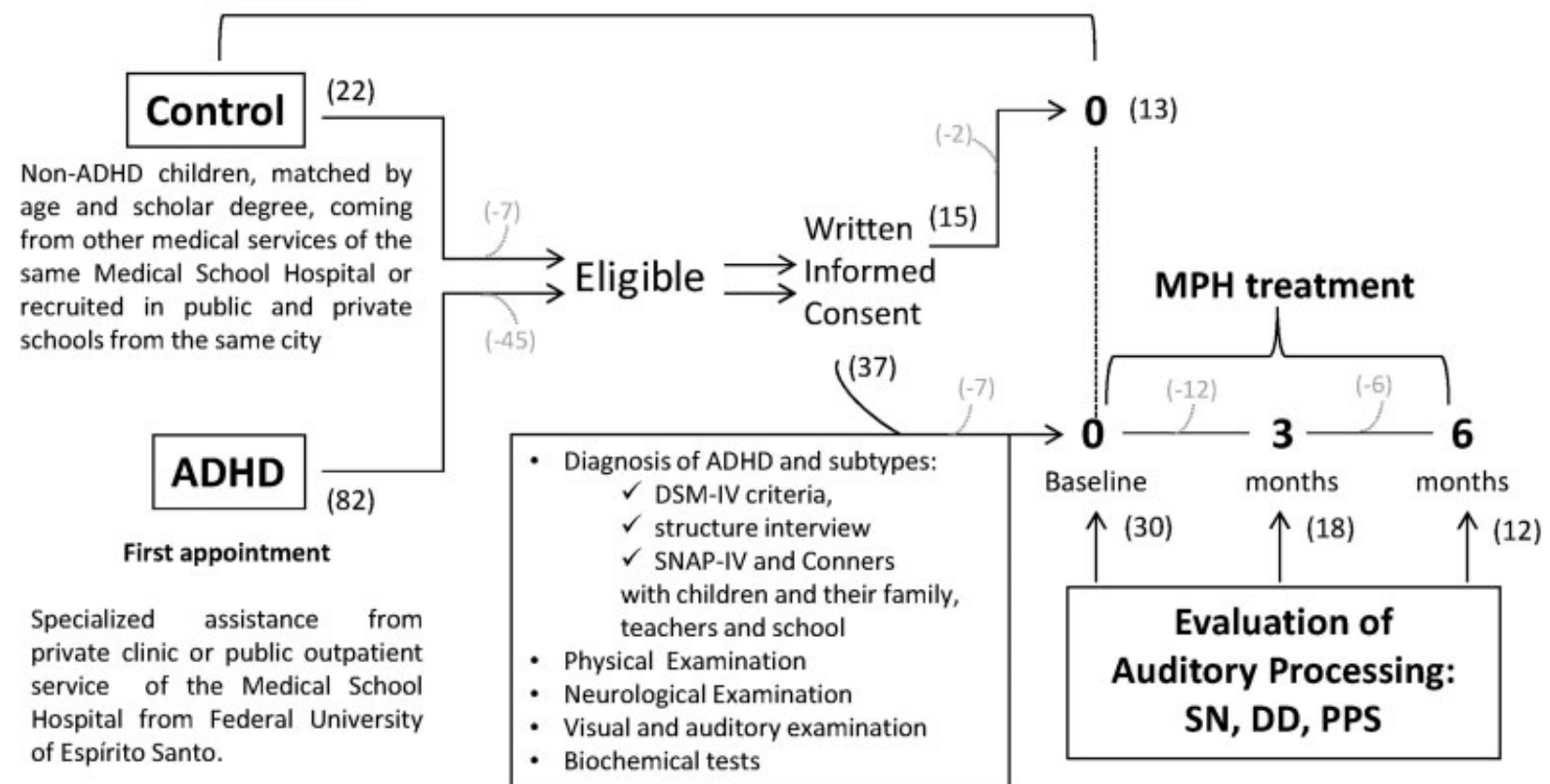

Fig. 1 Flow chart of the general experimental protocol. ADHD: Attention Deficit and Hyperactivity Disorder; DSM-IV: Diagnostic and Statistical Manual of Mental Disorders, Fourth Edition; SNAP-IV (Swanson et al., 2001); MPH: Methylphenidate (0.5 mg/kg/day); SN: speech with white noise; DD: dichotic digits; PPS: pitch pattern sequence.

auditory stimuli presented through standardized $\mathrm{CD}$ recording. The test consists of 30 randomized pattern sequences of three tones presented in each ear, yielding six different combinations of two frequencies ( 880 and $1430 \mathrm{~Hz}$ ). The stimuli were presented in the intensity of $50 \mathrm{~dB}$ HL above the average of the thresholds auditory frequencies. The children were asked to respond and classify verbally the sequence of stimuli and were evaluated by the number of correct responses.

\section{Statistical Analysis}

Data were presented by percentage or mean \pm standard deviation (SD) or percentage.

Two-sample unpaired $t$ test for counting parameters and Fisher for non-parametric data were used in comparisons between control and ADHD groups. A 2-tailed $\alpha$ level of 0.05 was used to determine statistical significance in these analyses.

The number of correct responses or errors obtained in the baseline (0), and after 3 and 6 months were expressed as mean \pm SD. For these analyses, we considered data from 18 ADHD children that entered to the first three-month follow-up examination. In the second three-month follow-up, completing 6 months of MPH treatment, missing data from 6 subjects were imputed by the last observation carried forward (LOCF) method allowing the intention-to-treat ${ }^{14}$ analysis to be performed. All data were analyzed with mixed-model analysis of variance ${ }^{15}$ considering two sides (left ear vs. right ear) as independent measures and three repeated measures $(0,3$, and 6 months), having correct responses for SN and PPS and number of errors for DD as dependent measures, followed by pair-wise $t$-test with Bonferroni's correction for multiple comparisons, thus considering a 2-tailed $\alpha$ level of 0.017 to determine statistical significance in these comparisons.

We used the GraphPad Prism 5.0 (GraphPad Software, Inc., San Diego, Calif.) and the Statistical Package for the Social Sciences (SPSS) software (SPSS, Inc., Chicago, Ill.) for statistical analysis and graphic presentations.

\section{Results}

From 82 children with probable ADHD diagnosis invited to the study, 37 were eligible ( 45 children were excluded because of learning disability, stuttering, incomplete diagnosis of ADHD, hearing loss, learning music, illiteracy, anxiety, dyslexia, use of antipsychotic medication) (-Fig. 1). From these 37 ADHD children, 7 were excluded because of hearing deficits, age above the limits by the end of the study and other clinic restrictions. Thus, 30 ADHD children were fully evaluated before the introduction of MPH. Out of these 30 ADHD children, 18 entered to auditory examination after 3 months of MPH treatment (12 were excluded for many reasons: none or low adherence to the medication, returning to the evaluations in wrong time, missing appointments), and finally 12 ADHD children completed the evaluation after 6 months of MPH treatment ( 6 were not included because of similar reasons mentioned above).

From 22 control children invited to this study, 15 accepted and 7 refused. Out of these 15, 2 were excluded because of 
hearing deficit and misunderstanding of auditory tasks (-Fig. 1).

\section{Socio-Demographic Characteristics}

There were no statistically significant differences between control $(n=13)$ and ADHD children $(n=30)$ regarding age, years of education or type of school attended (private versus public) (-Table 1). There was a higher proportion of males in the ADHD group (27M: $3 \mathrm{~F}$ ) compared with the control group (9M: 4F); however, Fisher's exact test showed no statistically significant difference between groups' distributions. There were also no differences of audiometric characteristics between groups ( - Table 1 ). All children showed normal audiometric curve, most of them were classified as type A in the tympanometry and presented acoustic reflexes.

\section{Auditory Processing}

\section{Non-ADHD versus ADHD Children}

ADHD children showed important differences when compared with matched control children regarding to auditory processing. They showed significantly less correct responses in the SN when tested in the left ear $(p=0.048)$, larger number of errors $(p=0.003)$ in both sides in the DD test, and much less correct responses $(p<0.0001)$ in the PPS test also in both sides.

Treatment with MPH in ADHD Children: 0, 3 and 6 Months There was no statistically significant difference in the SN between right and left ears $[\mathrm{F}(1,34)=0.16, p=0.69]$ and no significant interaction was found between the ear side and MPH effects $[F(1.6,54.9)=0.89, p=0.40]$ considering the Greenhouse-Geisser correction. A significant difference was found across 0,3 , and 6 month $S N$ examination $[F(1.6,54.9)=6.99$, $p=0.004]$ considering the Greenhouse-Geisser correction. We observed a greater number of correct responses after 6 months of MPH treatment in both sides, but it was statistically significant $\left[t^{17}=-3.06, p=0.007\right]$ in the left ear only when compared with the baseline scores (-Fig. 2 ).

No differences were found between right and left ears in the DD test $[\mathrm{F}(1,34)=0.02, p=0.89]$ and no significant interaction was found between the ear side and MPH effects $[F(1.4,46.3)=0.1, p=0.83]$ considering the GreenhouseGeisser correction. A significant difference was found across 0,3 , and 6 months of DD examination $[F(1.4,46.3)=20.57$, $p=0.000006$ ] considering the Greenhouse-Geisser correction. Significant smaller number of errors were found after 3 $\left[t^{17}=2.94, p=0.009\right]$ and $6\left[t^{17}=4.62, p=0.0002\right]$ months of MPH treatment in the right ear, and only after 6 months $\left[t^{17}=3.75, p=0.002\right]$ in the left ear when compared with the baseline scores (-Fig. $\mathbf{3}$ ).

There were no differences between right and left ears in the PPS test $[F(1,34)=1.39, p=0.25]$. Also, we found no interaction of the ear side over the MPH effects in this test $[\mathrm{F}(1.6,55.6)=0.28, p=0.71]$ considering the GreenhouseGeisser correction. However, there were significant differences across 0,3 , and 6 month of PPS examination $[\mathrm{F}(1.6,55.6)=14.09, p=0.00004]$ considering the Greenhouse-Geisser correction. Larger number of correct responses were found after 6 months of MPH treatment in the right $\left[t^{17}=-5.21, p=0.0007\right]$ and the left $\left[t^{17}=-3.08, p=0.007\right]$ ears when compared with the baseline scores (-Fig. 4).

\section{Discussion}

This study showed that at baseline, drug-naïve ADHD children showed poorer performance in auditory processing tasks when compared with age-gender-matched controls. The deficiencies improved significantly over 6 months of treatment with MPH.

Auditory processing mechanisms involve skills as auditory closure, binaural integration, and temporal ordering, all of which were impaired in the ADHD group before therapy. ${ }^{5}$

The differential diagnosis between ADHD and APD are complex, because decreased auditory discrimination in noisy environment or of competing sounds causes unsettling and distracted behaviors in both conditions. ${ }^{16}$ Since global attention deficits and low inhibitory control interfere in the $\mathrm{AP}$ assessment in $\mathrm{ADHD},{ }^{8}$ these disorders require a multidisciplinary approach to diagnosis. ${ }^{17}$

Our results evidenced abnormal left ear speech-in-noise test in ADHD children. This could suggest right hemisphere

Table 1 Auditory processing measurements in children with ADHD diagnosis and non-ADHD matched control

\begin{tabular}{|c|c|c|c|c|}
\hline & $\begin{array}{l}\text { Control group } \\
(n=13)\end{array}$ & $\begin{array}{l}\text { ADHD group } \\
(n=30)\end{array}$ & test & p-value \\
\hline $\begin{array}{l}\text { Mean } \pm \text { SD } \\
\text { SN (correct response) } \\
\text { Left } \\
\text { Right }\end{array}$ & $\begin{array}{l}20.2 \pm 1.3 \\
20.2 \pm 1.0\end{array}$ & $\begin{array}{l}18.8 \pm 2.2 \\
19.2 \pm 2.4\end{array}$ & $\begin{array}{l}t(41)=-2.04 \\
t(41)=-1.47\end{array}$ & $\begin{array}{l}0.048 \\
0.149\end{array}$ \\
\hline $\begin{array}{l}\text { DD (errors) } \\
\text { Left } \\
\text { Right }\end{array}$ & $\begin{array}{l}4.3 \pm 2.9 \\
4.3 \pm 3.2\end{array}$ & $\begin{array}{l}17.8 \pm 15.0 \\
17.6 \pm 14.9\end{array}$ & $\begin{array}{l}t(41)=3.20 \\
t(41)=3.17\end{array}$ & $\begin{array}{l}0.003 \\
0.003\end{array}$ \\
\hline $\begin{array}{l}\text { PPS (correct response) } \\
\text { Left } \\
\text { Right }\end{array}$ & $\begin{array}{l}27.6 \pm 2.3 \\
27.9 \pm 2.1\end{array}$ & $\begin{array}{l}18.4 \pm 6.4 \\
19.4 \pm 6.4\end{array}$ & $\begin{array}{l}t(41)=-5.06 \\
t(41)=-4.65\end{array}$ & $\begin{array}{l}<0.0001 \\
<0.0001\end{array}$ \\
\hline
\end{tabular}

Abbreviations: DD, dichotic digits; PPS, pitch pattern sequence; SN, speech with white noise. 


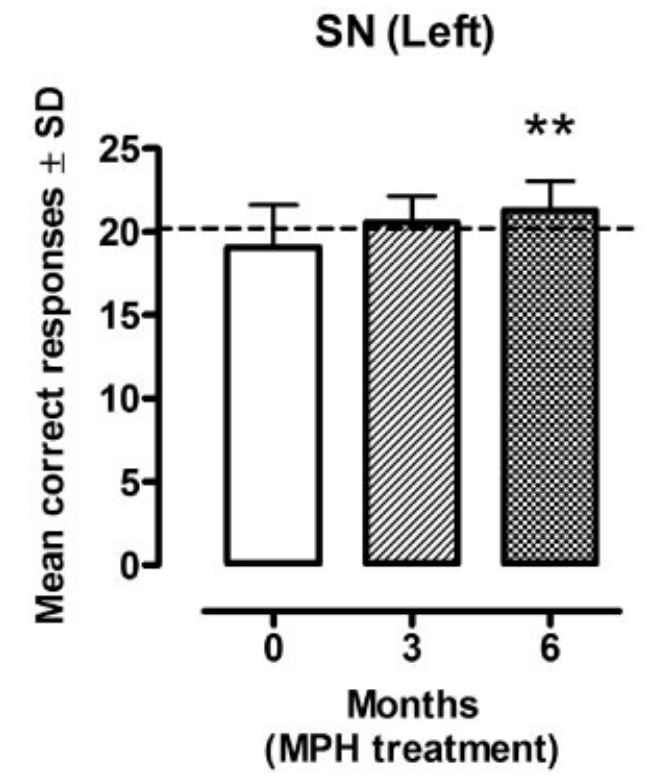

\section{SN (Right)}

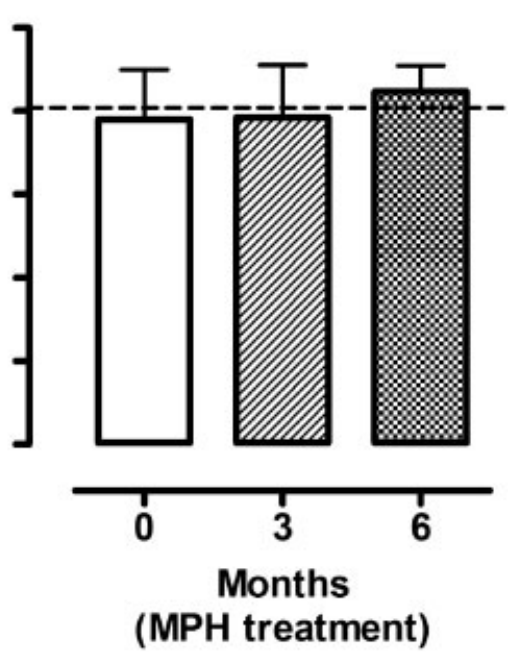

Fig. 2 Mean correct responses $( \pm S D)$ in the speech with white noise test $(S N)$ in children with diagnosis of Attention Deficit Hyperactive Disorder $(A D H D)(n=18)$ at baseline $(0)$, when naïve of treatment, and after 3 and 6 months of treatment with methylphenidate (MPH) in the left and right ears. ${ }^{* *} \mathrm{p}<0.01$ compared to the baseline. Traced line represents the mean of correct responses observed in matched non-ADHD children $(n=13)$.

DD (Left)

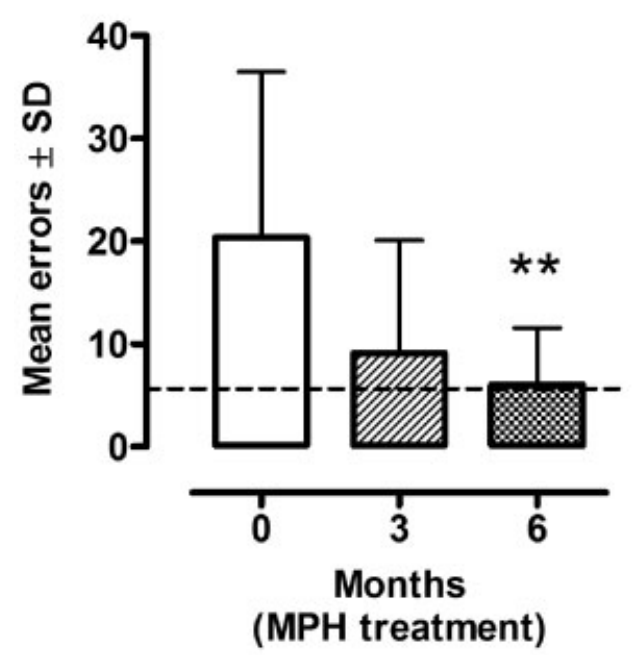

DD (Right)

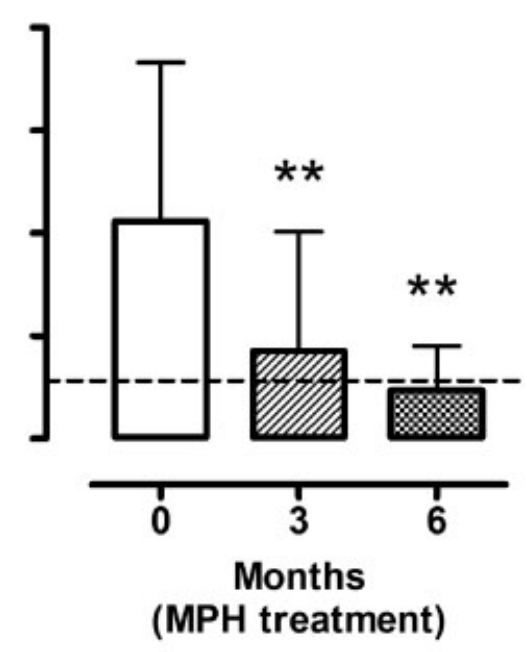

Fig. 3 Mean errors ( \pm SD) in the dichotic digits test (DD) in children with diagnosis of Attention Deficit Hyperactive Disorder (ADHD) $(n=18)$ at baseline $(0)$, when naïve of treatment, and after 3 and 6 months of treatment with methylphenidate (MPH) in the left and right ears. ${ }^{* *} \mathrm{p}<0.01$ compared to the baseline. Traced line represents the mean of correct responses observed in matched non-ADHD children $(n=13)$.

(RH) dysfunction and abnormal laterality. Impaired left-toright transfer of information, corpus callosum dysfunction, and delayed maturation of auditory nervous system may justify the left ear deficit, inadequate right ear advantage, and inconstant performance of ADHD group in AP evaluation. ${ }^{18}$ However, as we have not compared these results with other auditory processing tests, this hypothesis may not be fully concluded in this moment.

Positron emission tomography (PET) scanning studies reported higher activation of $\mathrm{RH}$ under non-verbal stimuli discrimination ${ }^{19}$ and temporal-order tasks ${ }^{13,20}$ in healthy participants. On the contrary, ADHD children presented time discrimination deficit and decreased activation of right fronto-cingulate region according to functional magnetic resonance imaging (fMRI) studies. ${ }^{21}$ Therefore, impaired PPS tests (evaluation of auditory temporal processing) in ADHD children compared with control children suggested time perception and evocation abnormality.

Previous studies reported controversial results regarding stimulant effects on auditory skills in ADHD 


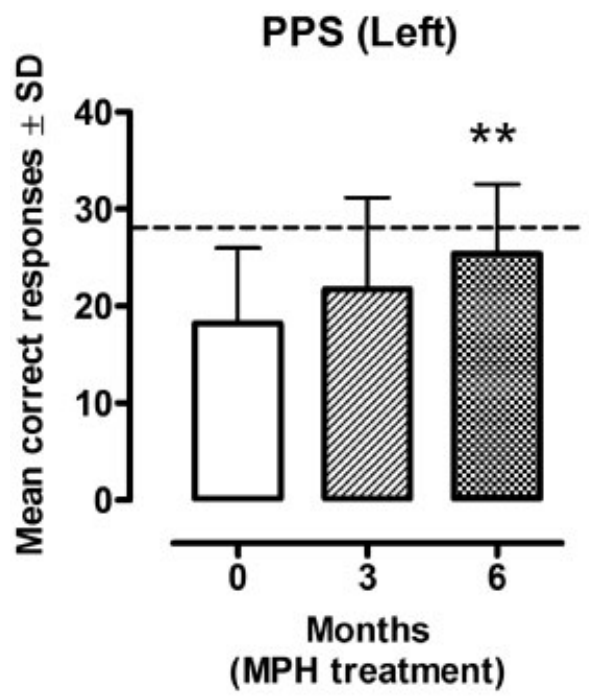

PPS (Right)

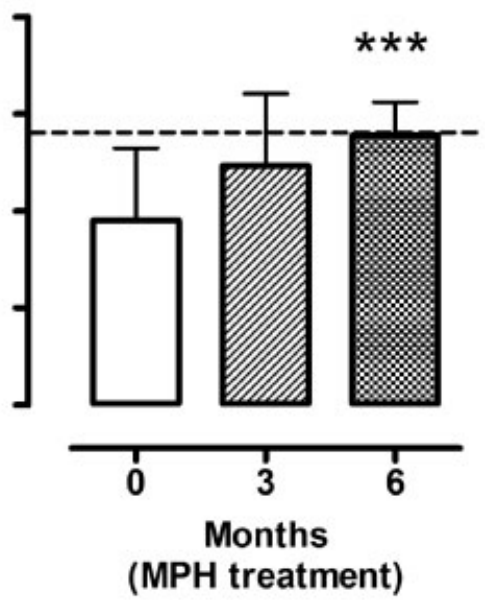

Fig. 4 Mean correct responses ( \pm SD) in the pitch pattern sequence test (PPS) in children with diagnosis of Attention Deficit Hyperactive Disorder $(A D H D)(n=18)$ at baseline (0), when naïve of treatment, and after 3 and 6 months of treatment with methylphenidate (MPH) in the left and right ears. ${ }^{* *} \mathrm{p}<0.01,{ }^{* * *} \mathrm{p}<0.001$ compared to the baseline. Traced line represents the mean of correct responses observed in matched non-ADHD children $(n=13)$.

children. Keith et $\mathrm{al}^{22}$ and Cook et $\mathrm{al}^{23}$ reported significant improvement of scores in auditory processing battery under pharmacological treatment when comparing ADHD children with and without AP dysfunction. By contrast, Tillery et al did not observe MPH effects other than behavioral attention improvement. ${ }^{24}$

In the current study, we observed a progressive improvement in the performance of AP tests, notably for DD and PPS tests, in ADHD children, reaching the most significant improvement at 6 months of MPH treatment. This progressive pattern of improvement produced by pharmacological treatment has been described for ADHD symptoms. ${ }^{25}$ However, for our best knowledge, this is the first evidence of progressive improvement produced by longtermed MPH treatment on auditory processing in ADHD children.

The study limitations should be mentioned. The small sample size and open label design may have underpowered this study and prevented a comparison between comorbid and non-comorbid groups with ADHD. However, the establishment of an untreated ADHD group was unviable due to ethical aspects. Besides, this study evoked unanswered questions about comorbid influences, MPH effects, and auditory processing performance in ADHD children. Confounding factors were minimized by using a control group matched for age, gender, and educational level. Unfortunately, we did not assess the effects of brain maturation over 6 month and the gender differences on auditory processing in ADHD children. However, Camarata and WoodCook ${ }^{26}$ followed a large cohort for 30 years to assess sex differences in cognitive abilities according to age in regular population and found no sex differences in auditory processing skills.

\section{Conclusion}

ADHD children show inefficient AP performance in selected behavioral auditory battery, suggesting impairment in auditory closure, binaural integration, and temporal ordering. Treatment with MPH gradually improved these deficiencies and completely reversed them by reaching similar scoring for control children at 6 months of treatment.

\section{References}

1 Diagnostic and Statistical Manual of Mental Disorders. 4th ed. Washington, DC: American Psychiatry Association; 2000

2 Polanczyk G, Rohde LA. Epidemiology of attention-deficit/hyperactivity disorder across the lifespan. Curr Opin Psychiatry 2007; 20(4):386-392

3 Willcutt EG. The prevalence of DSM-IV attention-deficit/hyperactivity disorder: a meta-analytic review. Neurotherapeutics 2012; 9(3):490-499

4 Jerger J, Musiek F. Report of the Consensus Conference on the Diagnosis of Auditory Processing Disorders in School-Aged Children. J Am Acad Audiol 2000;11(9):467-474

5 Dawes P, Bishop DV, Sirimanna T, Bamiou DE. Profile and aetiology of children diagnosed with auditory processing disorder (APD). Int J Pediatr Otorhinolaryngol 2008;72(4):483-489

6 Dawes P, Bishop D. Auditory processing disorder in relation to developmental disorders of language, communication and attention: a review and critique. Int J Lang Commun Disord 2009;44(4):440-465

7 Ghanizadeh A. Screening signs of auditory processing problem: does it distinguish attention deficit hyperactivity disorder subtypes in a clinical sample of children? Int J Pediatr Otorhinolaryngol 2009;73(1):81-87

8 Chermak GD. Differential Diagnosis of (Central) Auditory Processing Disorder and Attention Deficit and Hyperactivity Disorder. In: Musiek FEC, G.D., editor. Handbook of (Central) Auditory 
Processing Disorder. Auditory Neuroscience and Dignosis. 1. first ed. San Diego, CA, USA: Plural Publishing Inc.; 2007:365-94

9 Torgersen T, Gjervan B, Rasmussen K. Treatment of adult ADHD: is current knowledge useful to clinicians? Neuropsychiatr Dis Treat 2008;4(1):177-186

10 Ozdag MF, Yorbik O, Ulas UH, Hamamcioglu K, Vural O. Effect of methylphenidate on auditory event related potential in boys with attention deficit hyperactivity disorder. Int J Pediatr Otorhinolaryngol 2004;68(10):1267-1272

11 Swanson JM, Kraemer HC, Hinshaw SP, et al. Clinical relevance of the primary findings of the MTA: success rates based on severity of ADHD and ODD symptoms at the end of treatment. J Am Acad Child Adolesc Psychiatry 2001;40(2):168-179

12 Gaião AAB. An Epidemiological Study of Hyperkinetic Diusturbances: Diagnostic Norms and Validation of the Conners Questionnaire for Parents and Teachers. Infanto Rev Neuropsiquiatr Infanc Adolesc 1998;6(1):21-31

13 Pereira LD, Schochat E. Central Auditory Processing: Evoluation Manual. São Paulo, Brazil: Lovise; 1997

14 Jerger J, Thibodeau L, Martin J, et al. Behavioral and electrophysiologic evidence of auditory processing disorder: a twin study. J Am Acad Audiol 2002;13(8):438-460

15 Newcorn JH, Miller SR, Ivanova I, et al. Adolescent outcome of ADHD: impact of childhood conduct and anxiety disorders. CNS Spectr 2004;9(9):668-678

16 Geffner D. Language and Auditory Processing in ADHD. The ADHD Report. Special Issue Focus on Assess 2006;14(3):1-6

17 DiMaggio C. Prevalence of ADHD, speech and language delay, and familial factors associated with in children with CAPD. St. John's University.; 2003

18 Hale TS, Loo SK, Zaidel E, Hanada G, Macion J, Smalley SL. Rethinking a right hemisphere deficit in ADHD. J Atten Disord 2009;13(1):3-17
19 Mazziotta JC, Phelps ME, Meadors AK, Ricci A, Winter K, Bentson JR. Anatomical localization schemes for use in positron computed tomography using a specially designed headholder. J Comput Assist Tomogr 1982;6(4):848-853

20 Cabeza R, Anderson ND, Houle S, Mangels JA, Nyberg L. Age-related differences in neural activity during item and temporal-order memory retrieval: a positron emission tomography study. J Cogn Neurosci 2000;12(1):197-206

21 Smith AB, Taylor E, Brammer M, Halari R, Rubia K. Reduced activation in right lateral prefrontal cortex and anterior cingulate gyrus in medication-naïve adolescents with attention deficit hyperactivity disorder during time discrimination. J Child Psychol Psychiatry 2008;49(9):977-985

22 Keith RW, Engineer P. Effects of methylphenidate on the auditory processing abilities of children with attention deficit-hyperactivity disorder. J Learn Disabil 1991;24(10):630-636

23 Cook JR, Mausbach T, Burd L, et al. A preliminary study of the relationship between central auditory processing disorder and attention deficit disorder. J Psychiatry Neurosci 1993;18(3): 130-137

24 Tillery KL, Katz J, Keller WD. Effects of methylphenidate (Ritalin) on auditory performance in children with attention and auditory processing disorders. J Speech Lang Hear Res 2000;43(4):893-901

25 Brown RT, Amler RW, Freeman WS, et al; American Academy of Pediatrics Committee on Quality Improvement; American Academy of Pediatrics Subcommittee on Attention-Deficit/Hyperactivity Disorder. Treatment of attention-deficit/hyperactivity disorder: overview of the evidence. Pediatrics 2005;115(6): e749-e757

26 Camarata S, Woodcock R. Sex differences in processing speed: Developmental effects in males and females. Intelligence 2006; 34(3):231-252 